\title{
Partielles Ansprechen auf Crizotinib als Erstlinientherapie bei einem älteren männlichen Patienten mit ROS1-Translokations-positivem Bronchialkarzinom
}

\author{
Tobias R. Overbeck ${ }^{a, b} \quad K^{2}$ atja Schmitz ${ }^{c} \quad$ Christoph Engelke $^{d} \quad$ Carsten-Oliver Sahlmann $^{\text {e }}$ \\ Sara Hugo ${ }^{c}$ Laura Kellner ${ }^{c}$ Lorenz Trümper ${ }^{a, b}$ Hans-Ulrich Schildhaus ${ }^{a, c}$ \\ a Lungentumorzentrum Universität Göttingen and Göttingen Comprehensive Cancer Center (G-CCC), \\ ${ }^{b}$ Department of Hematology and Medical Oncology, \\ ' Institute of Pathology, \\ ${ }^{\mathrm{d}}$ Department of Radiology, and \\ e Department of Nuclear Medicine, University Hospital Göttingen, Göttingen, Germany
}

Schlüsselwörter

Präzisionsmedizin · Nicht-kleinzelliges Bronchialkarzinom .

ROS1-Rearrangement · Fluoreszenz-in-situ-Hybridisierung
ORIGINALARTIKEL FREI VERFÜGBAR UNTER

\section{Zusammenfassung}

Wir berichten hier über einen 90-jährigen männlichen Patienten mit ROS1-transloziertem Adenokarzinom der Lunge, der eine Erstlinientherapie mit Crizotinib erhielt. Nach 11-monatiger Behandlung waren ein vollständiges metabolisches Ansprechen laut ${ }^{18}$ F-FDG-PET/CT-Scan sowie ein partielles Ansprechen nach RECIST-Kriterien zu verzeichnen. Dieser Fall deutet darauf hin, dass ROS1-Translokationen sich nicht auf junges Alter, weibliches Ge- schlecht und niedrige Tumorstadien beschränken. Außerdem zeigt der Fall exemplarisch, dass die Crizotinib-Therapie auch als Erstlinientherapie bei älteren, komorbiden Patienten wirksam und beherrschbar sein kann. Auf Grundlage unserer Ergebnisse empfehlen wir, ältere Patienten mit fortgeschrittenem Adenokarzinom der Lunge in molekulare Screenings für ROS1-Translokationen einzuschließen.

\section{Einleitung}

Individualisierte Therapien im Sinne der personalisierten Medizin haben sich in der Behandlung von Bronchialkarzinomen als wirksam erwiesen [1]. Ein zur Aktivierung führendes Rearrangement der Rezeptortyrosinkinase ROS1 ist einer der molekularen Subtypen von Adenokarzinomen der Lunge [2], die Ansatzpunkte für eine zielgerichtete inhibierende Therapie bieten. Kürzlich konnte nachgewiesen werden, dass Crizotinib - ein kleines Molekül, das für die Behandlung von ALK-positivem
Lungenkrebs zugelassen ist - auch bei Bronchialkarzinomen mit ROS1-Translokationen Aktivität zeigt [3, 4]. Daher ist es wahrscheinlich, dass ein molekulares Screening auf ROS1-Translokationen in den meisten klinischen Settings eingeführt werden wird. Im Hinblick auf die Screeningmethoden sind Daten von Studienkohorten hervorzuheben, die darauf hindeuten, dass Patienten mit ROS1-Translokations-positiven Karzinomen deutlich jünger sind als solche mit ROS1-negativer Erkrankung [4-6].

\section{KARGER}

Fax +497614520714

information@karger.com

www.karger.com (c) 2016 S. Karger GmbH, Freiburg

Accessible online at:

www.karger.com/kko
Prof. Hans-Ulrich Schildhaus, MD

Institute of Pathology

University Hospital Göttingen

Robert-Koch-Strasse 40, 37075 Göttingen, Germany

hans-ulrich.schildhaus@med.uni-goettingen.de 

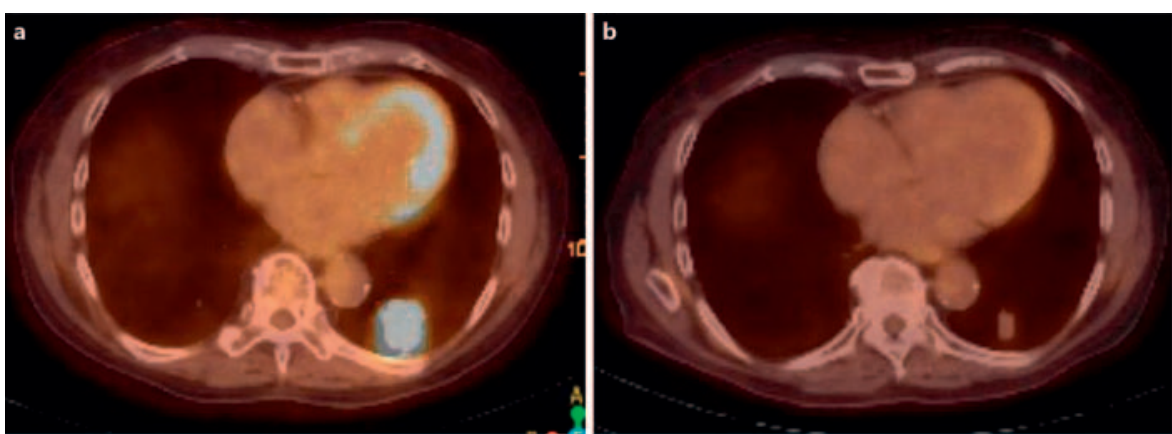

Abb. 1. Ansprechen auf die Behandlung. Primärtumor im linken Unterlappen. Vollständiges metabolisches Ansprechen auf Crizotinib laut 18F-FDG-PET/CT-Scans vor Behandlungsbeginn (a) und nach 4,5 Monaten Behandlung (b). Partielles Ansprechen gemäß RECIST-1.1-Kriterien: Tumorverkleinerung von $33 \mathrm{~mm}$ vor Behandlungsbeginn (c) auf $13 \mathrm{~mm}$ nach 4,5 Monaten Crizotinib (d)
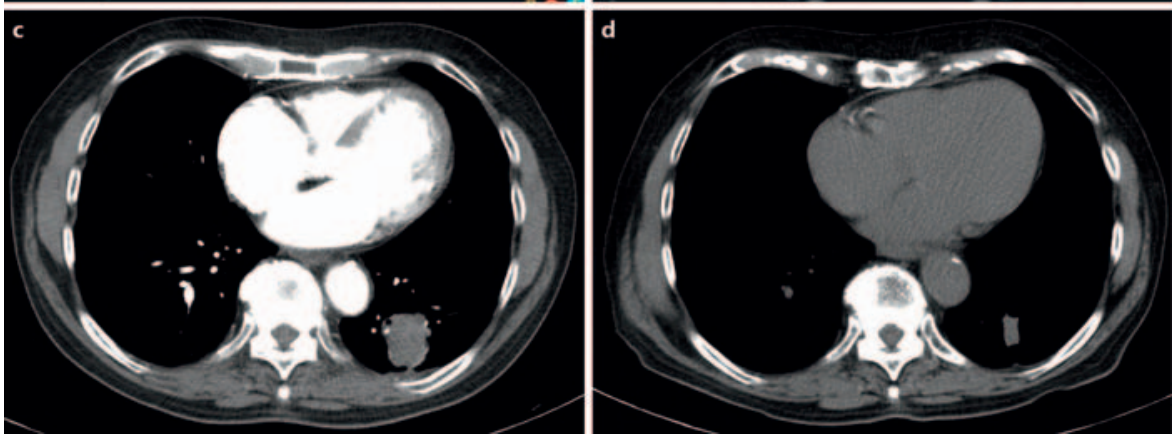

In einem kürzlich veröffentlichten Bericht über eine Population mit 72\% Personen nicht-asiatischer Abstammung mit ROS1Translokation betrug das mittlere Erkrankungsalter lediglich 49,8 Jahre und war damit signifikant niedriger als bei den ROS1negativen Patienten (mittleres Alter: 62,3 Jahre) und sogar niedriger als bei den Patienten mit ALK-transloziertem Karzinom (mittleres Alter: 51,6 Jahre) [7]. Deshalb wird vermutet, dass ein molekulares Screening auf ROS1-Translokationen auf jüngere Patienten zu beschränken wäre. Bei älteren Menschen besteht jedoch hoher klinischer Bedarf an maßgeschneiderten medikamentösen Therapieansätzen. Besonders dringend werden wirksame Erstlinientherapien benötigt, die rasch die Lebensqualität des Patienten wiederherstellen, da Patienten höheren Alters häufig an Komorbiditäten leiden, die ein konventionelles therapeutisches Vorgehen nicht erlauben.

\section{Vorstellung des Falls}

Ein 90-jähriger männlicher Patient stellte sich mit Dyspnoe in unserer Abteilung vor. Die Vorgeschichte umfasste koronare Herzkrankheit mit Herzinfarkt und doppelter koronarer Stentimplantation 4 Monate zuvor bei negativer Raucheranamnese. Weitere relevante Komorbiditäten waren Pericarditis epistenocardica und chronische Niereninsuffizienz. CT-Scans ergaben eine Raumforderung von 33 mm maximaler Größe im linken unteren Lungenlappen sowie Verdacht auf mediastinalen Lymphknotenbefall. In einem ${ }^{18}$ F-FDG-PET/CT-Scan stellte sich die primäre Läsion mit einem $S_{U} V_{\max }$ von 19,7 dar (Abb. 1). Darüber hinaus waren an Position 11L und 5 PET/CT-positive hiläre und mediastinale Lymphknoten zu erkennen. Dieser Befund führte zur Einstufung ins UICC-Stadium IIIA (cT2a, CN2, cM0). Der mediastinale Lymphknoten wurde durch eine endobronchiale Untersuchung nicht erreicht.
Eine Stanzbiopsie wurde entnommen. In der histologischen Untersuchung zeigte sich ein gering differenziertes Adenokarzinom vorwiegend vom soliden Subtyp. Immunohistochemische Untersuchungen auf Cytokeratin 7 und Thyroidalen Transkriptionsfaktor 1 fielen positiv aus, während Immunofärbungen für p63 und CD56 negativ blieben und so den Ausschluss eines plattenepithelialen oder neuroendokrinen Karzinom-Subtyps ermöglichten. Molekulare Tests auf EGFR- und KRAS-Mutationen sowie auf ALK-Translokation und MET-Amplifikation fielen sämtlich negativ aus. Eine FISH-Analyse (Fluoreszenz-in-situHybridisierung) auf ROS1-Translokation hingegen ergab aberrante Gensondensignale in 81 von 100 ausgewerteten Tumorzellkernen (Abb. 2). So konnte die Diagnose eines gering differenzierten pulmonalen Adenokarzinoms mit ROS1-Translokation gestellt werden.

Aufgrund des Tumorstadiums, der Komorbiditäten und des Alters kam eine Operation oder konventionelle Chemotherapie für den Patienten nicht in Frage. Eine stereotaktische Strahlentherapie der primären Läsion wurde diskutiert; aufgrund des molekularen Befundes einer ROS1-Translokation fiel jedoch die Entscheidung dagegen. Nach Besprechung der Tumortests und Aufklärungsgespräch wurde der Patient mit Crizotinib in einer Dosierung von zunächst $250 \mathrm{mg}$ p.o. zweimal täglich behandelt. Die Laborwerte von Tag 7 zeigten einen weiteren Anstieg der bereits zuvor erhöhten Gamma-Glutamyl-Transpeptidase (GGT) auf das Zwei- bis Vierfache der Obergrenze des Normbereichs (UNL; upper normal limit) sowie eine Erhöhung der Glutamat-Oxalacetat-Transaminase (GOT) und der AspartatAminotransferase (ASAT) von normalen Werten auf das Zweifache des UNL. Im gleichen Zeitraum stieg der Serumkreatininspiegel von 1,6 auf 2,0 mg/dl. Nach 7-tägiger Pause wurde die Behandlung mit Crizotinib mit einer reduzierten Dosis von $250 \mathrm{mg}$ täglich wieder aufgenommen, nachdem sich die Labor- 
Abb. 2. Histologischer und molekularer Befund. a Solides Adenokarzinom der Lunge (HE-Färbung). b ROS1-Translokation, nachgewiesen mittels FISH. Grüne Sonden flankieren das 3'-Ende des ROS1-Gens; normale Zellen zeigen orange-grüne Fusionssignale. Gen-Rearrangements sind erkennbar an entweder isolierten grünen Signalen oder getrennten orangefarbenen und grünen Break-Apart-Signalen oder einer Kombination aus beiden Mustern in $\geq 15 \%$ der Tumorzellen (Sonde zur Verfügung gestellt von ZytoVision, Bremerhaven, Deutschland).
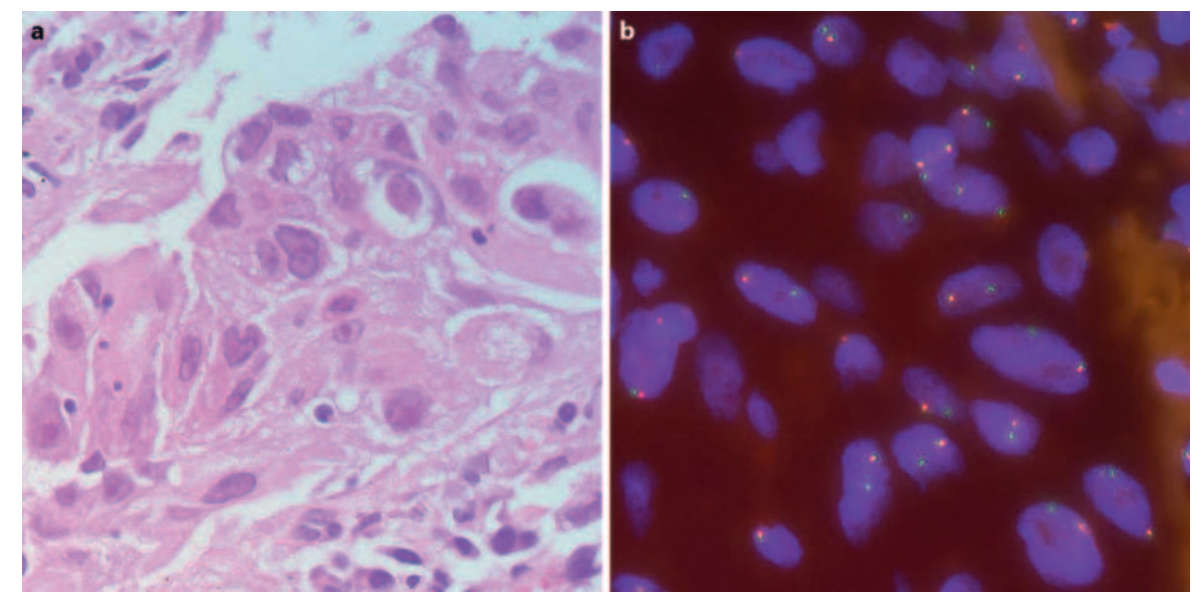

werte verbessert hatten, wobei der Serumkreatininwert auf dem vorherigen erhöhten Niveau verblieb. Der Patient klagte über Beschwerden wie Übelkeit, Erbrechen und Diarrhö ab dem 5. Tag nach der Dosissenkung. An Tag 15 der Behandlung mit Crizotinib $250 \mathrm{mg}$ p.o. einmal täglich traten Schmerzen im Brustraum auf, woraufhin die Medikation abgesetzt wurde. Mit einer Verzögerung von 3,5 Wochen wurde die Behandlung mit Crizotinib $250 \mathrm{mg}$ einmal täglich wieder aufgenommen. Eine akute ulzerierende, fibrinöse, eitrige Ösophagitis, die nach 4,5 Monaten auftrat, klang unter Behandlung mit oralen H2-Blockern wieder ab. Zusammengefasst waren unerwünschte Ereignisse über einen Zeitraum von 11 Monaten für behandlungsfreie Intervalle von insgesamt 11,5 Wochen verantwortlich. Die erste Tumor-Evaluierung erfolgte mittels Thoraxröntgen 2 und 3 Monate nach Behandlungsbeginn mit Crizotinib. Die planaren Maße der primären Läsion gingen von $26 \times 50 \mathrm{~mm}$ bei Behandlungsbeginn auf $18 \times 42$ bzw. $13 \times 34 \mathrm{~mm}$ zurück. CT-Scans nach 4,5 Monaten zeigen eine partielle Remission der primären Läsion von 33 auf $13 \mathrm{~mm}(\mathrm{CT})$ und ein vollständiges metabolisches Ansprechen (PET). Zu diesem Zeitpunkt waren die hilären und mediastinalen Lymphknoten im CT- und PET-Scan noch vorhanden. Röntgenaufnahmen des Brustraums nach 7 und 10 Monaten zeigten keine bedeutenden Veränderungen. Nach 11 Monaten wird der Patient weiterhin mit Crizotinib $250 \mathrm{mg}$ täglich behandelt (d.h. mit der Hälfte der empfohlenen Dosis), ohne jede Einschränkung im täglichen Leben und mit einem ECOG-Performance-Status von 0.

\section{Diskussion}

ROS1-Translokationen liegen bei 1,8\% der pulmonalen Adenokarzinome vor, unabhängig vom Tumorstadium und Malignitätsgrad (persönliche Beobachtung). Diese Häufigkeit ergibt eine geschätzte Gesamtzahl von 2500-3300 Fällen ROS1-positiver Bronchialkarzinome in den USA im Jahr 2015. Aktuelle Daten liefern Belege dafür, dass Crizotinib, ein TKI mit Aktivität gegen MET, ALK, RON und ROS1, bei Patienten mit ROS1-transloziertem Bronchialkarzinom hochaktiv ist $[3,4]$. Es wurde beobachtet, dass die Behandlung zu dauerhafter, langanhaltender Tu- morverkleinerung und guter klinischer Symptombeherrschung führte.

Alle bisherigen klinischen Berichte zu ROS1-Veränderungen bei Lungenkrebs betonen jedoch, dass ROS1-assoziierte Adenokarzinome lediglich auf frühere klinische Stadien, weibliches Geschlecht und jüngeres Lebensalter beschränkt seien. ROS1-Translokationen sind bei Patienten im Alter von 25 bis 77 Jahren nachgewiesen worden; das Durchschnittsalter war hierbei noch niedriger als bei Patienten mit ALK-positiven Bronchialkarzinomen $[4,7]$.

Wir berichten hier von einem männlichen Patienten höheren Alters mit fortgeschrittenem ROS1-positivem Adenokarzinom der Lunge, der unter TKI-Therapie metabolisches Ansprechen und signifikante Tumorverkleinerung zeigte. Ein partielles Ansprechen nach RECIST-1.1-Kriterien wurde innerhalb von 4,5 Monaten erreicht. Wie es bei Menschen höheren Alters häufig der Fall ist, war unser Patient aufgrund der Komorbidität kein Kandidat für die standardmäßigen Therapieschemata. Im Vergleich zu konventionellen Chemo- oder Radiochemotherapien ist Crizotinib vom Sicherheitsprofil her für viele Patienten mit Komorbiditäten geeignet; schwerwiegende Nebenwirkungen wurden bisher nur selten beschrieben $[4,8]$. In unserem Fall war die CrizotinibTherapie klinisch beherrschbar. Erhöhte Transaminasewerte und klinische Beschwerden machten Behandlungspausen und eine Dosisreduktion erforderlich. Das klinische Ansprechen wurde jedoch auch mit der verringerten Dosis von $250 \mathrm{mg}$ Crizotinib einmal täglich erreicht. Und was am wichtigsten ist: Die allgemeine Lebensqualität konnte rasch wiederhergestellt werden, und stationäre Behandlungen sowie toxische Chemotherapien blieben dem Patienten während der gesamten Therapie erspart.

Da wir ein gutes Ansprechen auf eine TKI-Therapie antizipierten, entschieden wir uns für die Anwendung von Crizotinib gleich als Erstlinientherapie. Bisher liegen jedoch nur in begrenztem Umfang Daten zu diesem Arzneimittel in der ersten Therapielinie bei ROS1-translozierten Tumoren vor. Unseres Wissens liegen bisher erst Berichte zu 7 Patienten vor [4], die alle auf die Behandlung ansprachen. Diese Daten und unsere Ergebnisse könnten gemeinsam eine Rationale für Crizotinib zur Erstlinienbehandlung ROS1-positiver Tumoren im Rahmen klinischer Studien liefern. 
Unser Fall zeigt, dass für schnelle klinische Entscheidungen über Therapieoptionen die schnelle Bereitstellung von Ergebnissen aus dem molekularen Screening erforderlich ist. Daher empfehlen wir die Nutzung von molekularen Panels als Reflextest. Für den Nachweis von ROS1-Translokationen scheint die FISH-Analyse am besten geeignet zu sein, da die Ergebnisse schon 2 Tage nach der ersten Diagnosestellung vorliegen. Es wurde nachgewiesen, dass keine Korrelation zwischen der Art der Translokation, d.h. dem spezifischen Translokationspartner von ROS1, und dem Ansprechen auf Crizotinib besteht [4]. Dass die FISH-Analyse alle Unterarten von ROS1-Rearrangements erkennt, rechtfertigt zusätzlich, warum dieses Verfahren derzeit das am besten geeignete für den Nachweis von ROS1-Veränderungen ist.

Zusammenfassend lässt sich sagen, dass unser Bericht über den bisher ältesten Patienten mit ROS1-positivem Bronchialkarzinom darauf hindeutet, dass ROS1-Translokationen sich nicht auf junge Patienten weiblichen Geschlechts in niedrigen Tumorstadien beschränken. Unser Fall zeigt exemplarisch, dass Crizotinib auch als Erstlinientherapie bei älteren, komorbiden Patienten wirksam und durchführbar sein kann. Auf der Grundlage unserer Ergeb- nisse empfehlen wir, ältere Patienten mit fortgeschrittenem Adenokarzinom der Lunge in molekulare Screenings für ROS1Translokationen einzuschließen.

\section{Danksagung}

Die Autoren danken dem Patienten, der der Veröffentlichung dieses Berichts zugestimmt hat.

\section{Erklärung zu ethischen Konflikten}

Die Veröffentlichung wurde durch den Patienten genehmigt.

\section{Disclosure Statement}

H.U.S. hat Forschungsförderungen von Novartis Oncology und ZytoVision (außerhalb dieser Arbeit) erhalten, außerdem Honorare und Vergütungen für Beiratsmitgliedschaft und Vorträge von Pfizer, Novartis, Roche, Zytomed, ZytoVision und Abbott Molecular. T.O. hat Honorare und Vergütungen für Beiratsmitgliedschaft und Vorträge von Astra Zeneca, BMS, Boehringer Ingelheim, Clovis Oncology, Lilly, medac, MSD, Novartis, sanofi-aventis und Roche erhalten.

\section{Referenzen}

$>1$ Clinical Lung Cancer Genome Project (CLCGP); Network Genomic Medicine (NGM): A genomics-based classification of human lung tumors. Sci Transl Med 2013;5:209ra153.

-2 Rikova K, Guo A, Zeng Q, et al: Global survey of phosphotyrosine signaling identifies oncogenic kinases in lung cancer. Cell 2007;131: 1190-1203.

3 Bos M, Gardizi M, Schildhaus HU, et al: Complete metabolic response in a patient with repeatedly relapsed non-small cell lung cancer harboring ROS1 gene rearrangement after treatment with crizotinib. Lung Cancer 2013; 81:142-143.
4 Shaw AT, Ou S-HI, Bang Y-J, et al: Crizotinib in ROS1-rearranged non-small-cell lung cancer. New Engl J Med 2014;371:1963-1971.

5 Chen Y-F, Hsieh M-S, Wu S-G, et al: Clinical and the prognostic characteristics of lung adenocarcinoma patients with ROS1 fusion in comparison with other driver mutations in East Asian populations. J Thorac Oncol 2014; 9:1171-1179.

6 Yoshida A, Kohno T, Tsuta K, et al: ROS1-rearranged lung cancer: a clinicopathologic and molecular study of 15 surgical cases. Am J Surg Pathol 2013;37:554-562.
Bergethon K, Shaw AT, Ignatius Ou S-H, et al: ROS1 rearrangements define a unique molecular class of lung cancers. J Clin Oncol 2012;30:863-870.

8 Ono A, Takahashi T, Oishi T, et al: Acute lung injury with alveolar hemorrhage as adverse drug reaction related to crizotinib. J Clin Oncol 2013;31:e417-e419. 